\title{
Studies on Yttrium Incorporated Hydroxyapatite Nanocrystals Prepared by Sol Gel Method
}

\author{
Saranya $\mathrm{S}^{1}$, Devi $\mathrm{G}^{2}$, Kavitha $\mathrm{V}^{3}$,Prema Rani $\mathrm{M}^{4}$ \\ \{saranyaashok122012@gmail.com ${ }^{1}$,mailengr.devi@gmail.com², premaakumar91@gmail.com ${ }^{3}$, \\ kaviram06@gmail.com ${ }^{4}$ \} \\ ${ }^{1}$ Department of Physics, S.V.N College, Madurai, Tamilnadu, India \\ ${ }^{2}$ Department of EEE, PSNA College of Engineering and Technology, Dindigul, Tamilnadu, India \\ ${ }^{3,4}$ Department of Physics, Madura College, Madurai, Tamilnadu, India
}

\begin{abstract}
Yttrium doped hydroxyapatite nanocrystals were synthesized by sol gel method for three different concentrations of yttrium $(3 \%, 6 \% \& 9 \%)$. XRD was used to examine structural parameters, SEM was used to examine surface morphology, and EDS was used to determine the elemental composition. XRD revealed that the crystallite size was $28 \mathrm{~nm}$. When XRD data is compared to JCPDS data, the strength peaks from XRD data are found to be similar. Space group of the grown samples has been found to be $\mathrm{P} 63 / \mathrm{m}$. From the FTIR analysis the presence of HPO4 -was confirmed which reinforces that HAP was synthesized.
\end{abstract}

Keywords: Crystallite Size, Doping, FTIR, Hydroxyapatite, XRD.

\section{Introduction}

$\left(\mathrm{Ca}_{5}(\mathrm{PO} 4)_{3} \mathrm{OH}\right)$ is a biomaterial with a diverse set of uses. It's also known as bone mineral because it crystallises in a hexagonal system. It's filler that's used to patch amputated bone [1]. HAP is used to coat modern implants such as hip replacements, dental implants, and bone conduction implants $[2,3]$. Porous HAP is a form of HAP that is used to deliver drugs to specific areas of the body, such as the bones $[4,5]$.

HAP has a low host reaction. When metal ions are doped in HAP, catalytic properties are observed [6, 7].Antibacterial activity is observed when yttrium is doped in HAP [8]. It slows bacterial growth in the mouth slightly. Human periodontal fibroblast development is accelerated by it [9]. It attracts water molecules with a high affinity. It boosts the performance of HaP-cultured cells [10]. It makes the proton environment more favourable.

\section{Experimental Procedure}

Solgel was used to make yttrium doped hydroxyapatite nanocrystals. In deionized water, diammonium hydrogen phosphate was dissolved and stirred at $90^{\circ} \mathrm{C}$ in a magnetic stirrer. Separately dissolved calcium nitrate tetrahydrate and Yttrium nitrate were added to deionized water. Drop by drop, the above two solutions were applied. Ammonium hydroxide was used 
to keep the $\mathrm{P}_{\mathrm{H}}$ at 10 . For six hours, the solution was held at $90^{\circ} \mathrm{C}$. The precipitates were filtered and washed before being dried for 22 hours at $110^{\circ} \mathrm{C}$ and then calcined for 2 hours at $700^{\circ} \mathrm{C}$.

\section{Characterization}

\subsection{XRD}

The phases of the samples were identified by powder X-Ray diffraction done at SAIF, Cochin with $\mathrm{Cu} K \alpha(\lambda=1.5406 \mathrm{~A} 0)$. The XRD data set was obtained using two ranges ranging from 100 to 1200 with a 0.020 phase scale. The obtained data was compared with JCPDS and crystal structure was confirmed as hexagonal with space group of p63/m. shows the XRD profiles of pure and doped hydroxyapatite samples. Table 1 shows the crystallite size as determined by XRD. The relationship between 2 and crystallite size is depicted in Figure 2 .

Table 1. Crystallite size from XRD

\begin{tabular}{|l|l|l|l|}
\hline S.No & Sample & $2 \theta$ in degrees & Crystallite size in $\mathrm{nm}$ \\
\hline 1 & Pure HAP & 31.796 & 28.1205 \\
\hline 2 & 3\%Y doped HAP & 31.807 & 28.1115 \\
\hline 3 & $6 \%$ Y doped HAP & 31.836 & 28.0864 \\
\hline 4 & $9 \%$ doped HAP & 31.858 & 28.0676 \\
\hline
\end{tabular}

The size of crystallites was affected by yttrium doping. If the concentration of yttrium rises, the size of the crystallite shrinks. Because the ionic radius of yttrium $(0.09 \mathrm{~nm})$ is smaller than that of calcium, this proves that $\mathrm{Y}^{3+}$ ions replace $\mathrm{Ca}^{3+}$ ions in the HAP. $(0.1 \mathrm{~nm})$. Bragg's law states that as the interplanar distance decreases, the lattice parameter decreases. This also shows that as the concentration of $\mathrm{Y}$ increases, the crystallite size decreases.

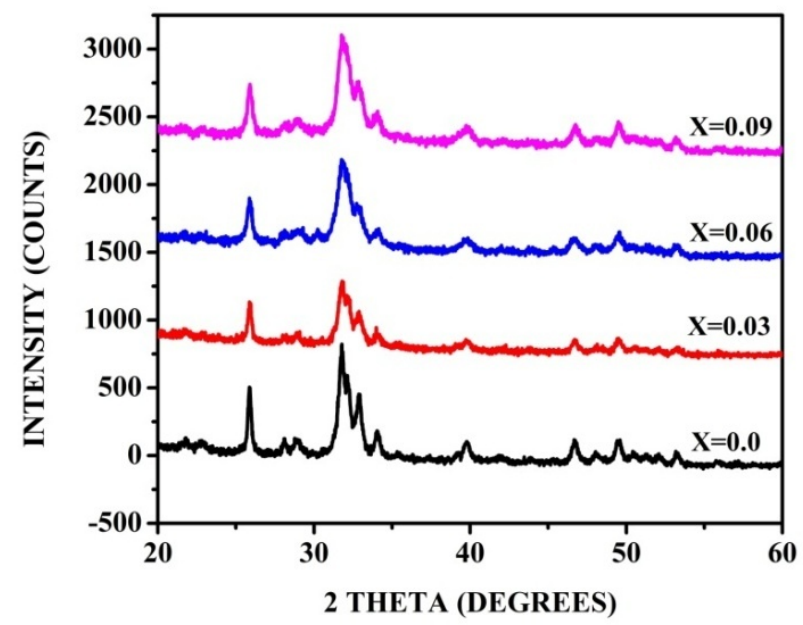

Fig. 1. XRD profiles for the pure and Yttrium doped Hydroxyapatite 


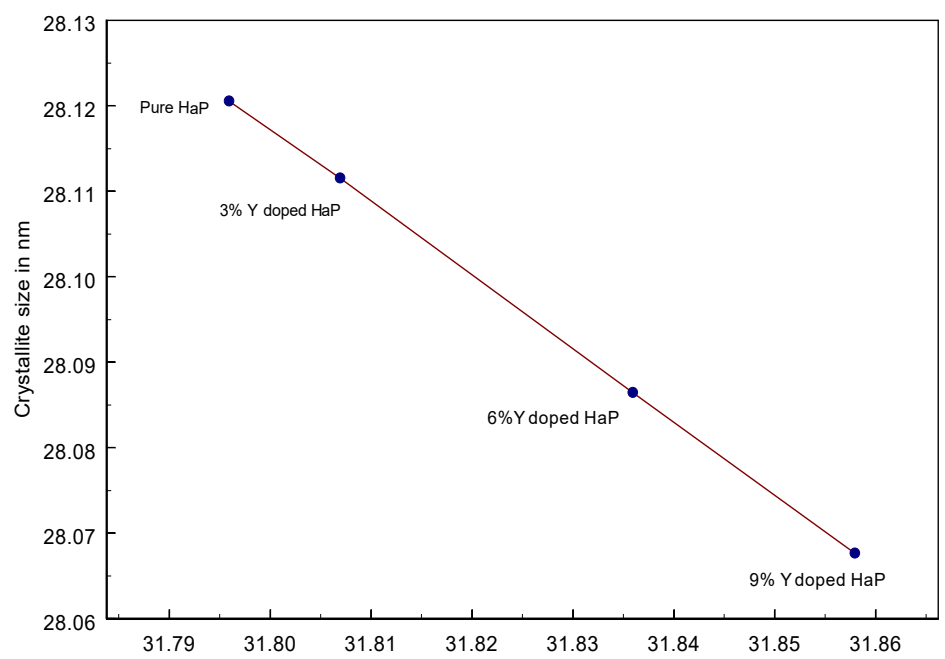

Fig. 2. Relation between $2 \theta$ and crystallite size of pure and Yttrium doped Hydroxyapatite

\subsection{FTIR}

Fourier transform infrared spectroscopy was used to validate the existence of functional groups. The spectrum was captured in the $4000-400 \mathrm{~cm}-1$ range. The spectrometer's resolution was $4 \mathrm{~cm}-1$. Many of HAP absorption peaks can be seen in FTIR spectra. Spectra were collected in the 472.56-3570.23 area. FTIR profiles of pure and doped Hydroxyapatite are shown in Figures 3a-3d. The FTIR peaks and their inferences are mentioned in Table 2.

Table 2. Peaks from FTIR and their corresponding inference

\begin{tabular}{|l|l|l|l|l|l|}
\hline $\begin{array}{l}\text { Sl. } \\
\text { No. }\end{array}$ & $\begin{array}{l}\text { Chemical } \\
\text { group }\end{array}$ & $\begin{array}{l}\text { pure } \\
\text { hap }\end{array}$ & $\begin{array}{l}3 \% \mathrm{Y} \text { doped } \\
\text { hap }\end{array}$ & $\begin{array}{l}6 \% \mathrm{Y} \\
\text { doped } \\
\text { hap }\end{array}$ & $\begin{array}{l}9 \% \mathrm{Y} \text { doped } \\
\text { hap }\end{array}$ \\
\hline 1 & $\mathrm{OH}^{-}$ & 3423.65 & 3417.86 & 3514.93 & - \\
\hline 2 & $\mathrm{CO}_{3}^{2-}$ & 1411.89 & 1413.82 & 1413.92 & 1415.75 \\
\hline 3 & $\mathrm{Adsorbed} \mathrm{water}^{2-}$ & - & 2829.57 & 2833.43 & 2835.36 \\
\hline 4 & $\mathrm{HPO}_{4}^{2-}$ & - & 877.61 & 876.61 & 875.68 \\
\hline 5 & $\mathrm{PO}_{4}^{3-}$ & 601.79 & 603.72 & 603.72 & 603.72 \\
\hline
\end{tabular}

Fourier transform-infrared spectroscopy is an analytical technique that uses the absorption or emission spectrum of materials to distinguish organic and inorganic materials. The existence of HAP is confirmed by FTIR performance. With a rise in yttrium concentration, we expect a decrease in the IR absorption bands of the phosphate groups of the samples. 


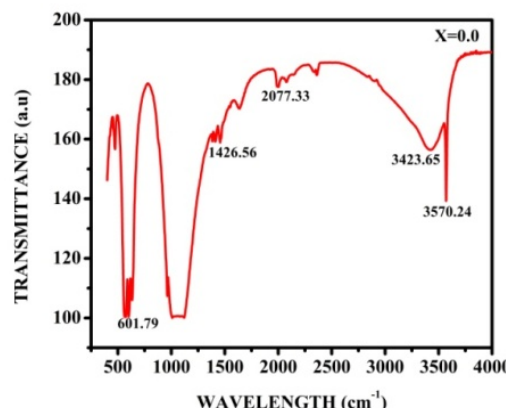

3(a)

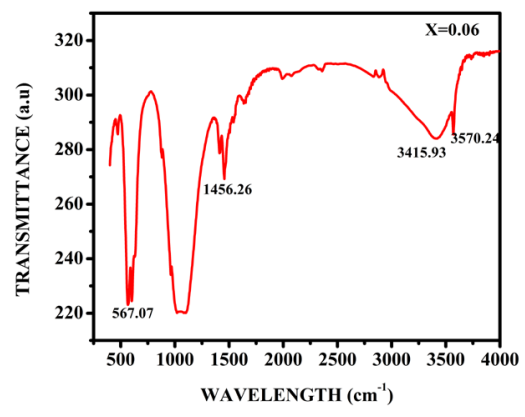

$3(\mathrm{c})$

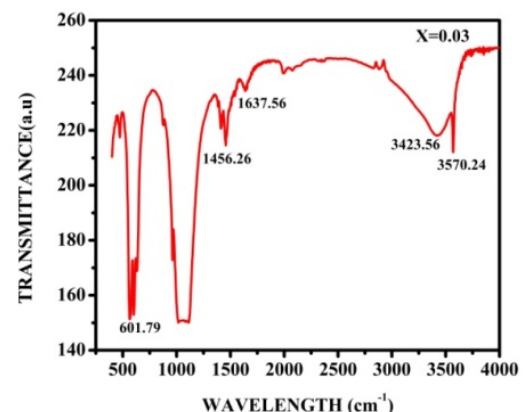

$3(\mathrm{~b})$

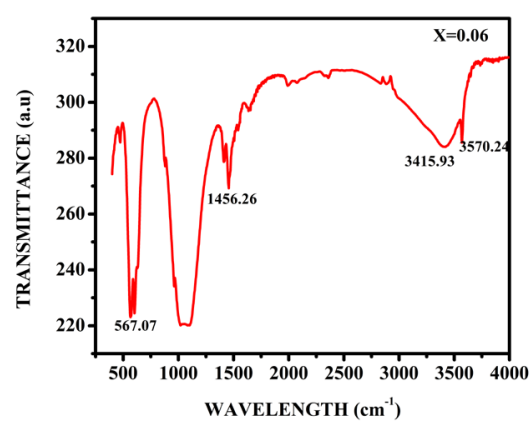

$3(d)$

Fig. 3. FTIR profiles for the pure and doped Hydroxyapatite

\subsection{SEM}

SEM images of the samples were taken at International Research Centre, Kalasalingam University, Krishnan kovil. Figure 4 represents the SEM images of pure and doped hydroxyapatite. 

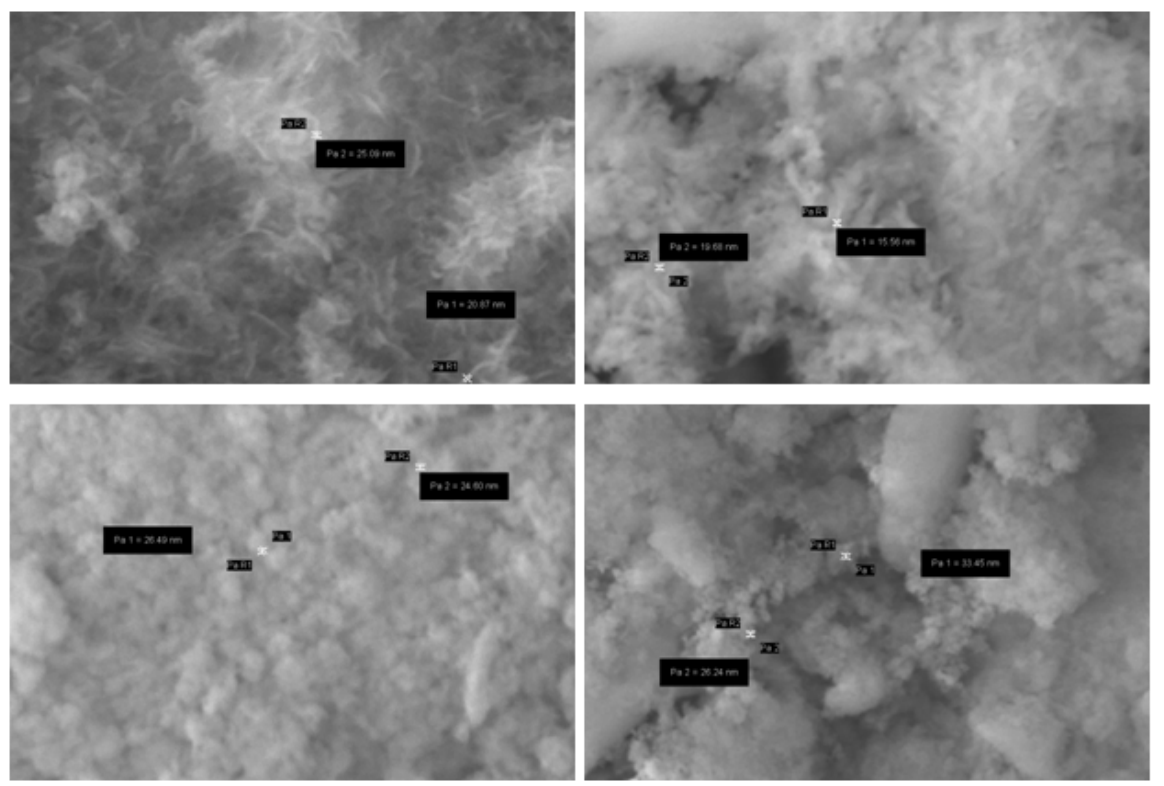

Fig. 4. SEM images of pure and doped Hydroxyapatite

\subsection{EDS}

Gandhigram Rural University performed the chemical characterizations of the samples. EDS representations of pure and doped hydroxyapatite are depicted in Figures 5a-5d. In pure and doped Hydroxyapatite, Table 3 shows the constituent elements.

Table 3. Constituent elements of pure and doped HAP

\begin{tabular}{|l|l|l|l|l|l|}
\hline Sl. No. & Element & Pure HAP & 3\% doped HAP & 6\% doped HAP & 9\% doped HAP \\
\hline 1 & $\mathrm{O}$ & 38.81 & 62.05 & 42.24 & 14.94 \\
\hline 2 & $\mathrm{C}$ & - & 14.76 & 15.23 & 24.68 \\
\hline 3 & $\mathrm{Ca}$ & 42.26 & 20.12 & 18.27 & 2.67 \\
\hline 4 & $\mathrm{P}$ & 16.32 & 10.03 & 9.57 & 5.55 \\
\hline 5 & $\mathrm{Y}$ & - & 1.06 & 5.62 & 3.32 \\
\hline
\end{tabular}



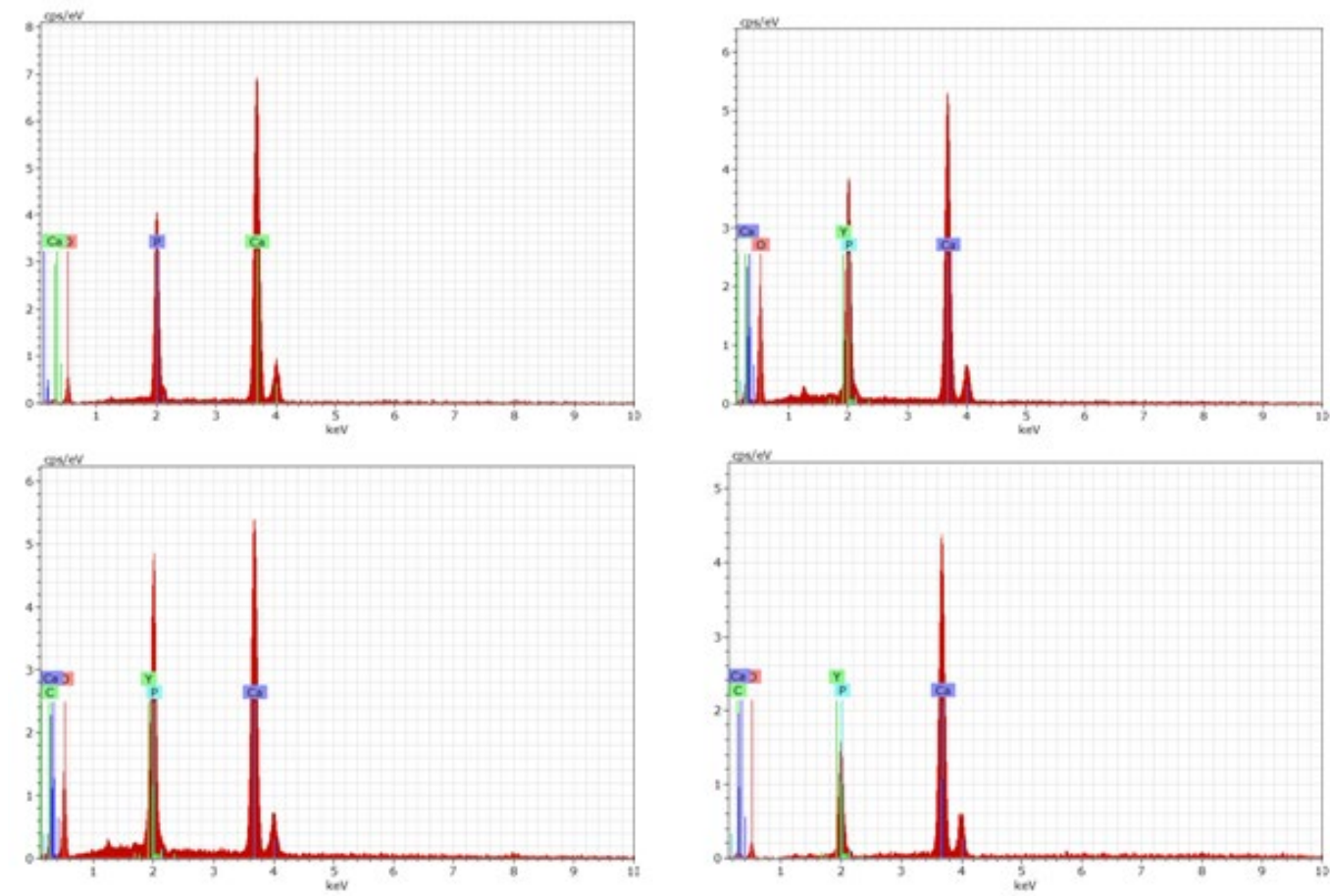

Fig. 5. EDS images of pure and doped Hydroxyapatite

\section{Conclusion}

The sol gel method was used to successfully synthesise yttrium doped hydroxyapatite nanocrystals in three different concentrations of yttrium ( 3 percent , 6 percent \& 9 percent ). The crystallite size was measured using XRD, and the crystallite size decreased as the Yttrium concentration increased. Using XRD data and JCPDS files as P63/m, the space category was calculated. The existence of an HPO4- group with a wavenumber in the order of $870 \mathrm{~cm}-1$ was confirmed by FTIR. SEM was used to examine the surface morphology, and EDS was used to determine the elemental composition.

\section{References}

[1] Setsuaki Murakami,Katsuya Kato, Yuki Enari, Masanobu Kamitakahara, Noriaki Watanabe, and KojiIoku,Hydrothermal synthesis of porous hydroxyapatite ceramics composed of rod-shaped particles and evaluation of their fracturebehavior, Ceramics international, 38(2), 2012, 1649-1654.

[2] Yan Li, Dandan Wang and Sierin Lim,Fabrication and Applications of Metal-Ion Doped Hydroxyapatite Nanoparticles, Juniper Online Journal Material Science, 1(2), 2017.

[3] Alek Sandra Sccze's, Lucyna Holysz and Emil Chibowski, Synthesis of HaP for biomedical applications, Advances in Colloid and Interface Science, Volume 249, November 2017, 321-330.

[4] Wei G, Ma PX, Structure and properties of nano-hydroxyapatite/polymer composite scaffolds for bone tissue engineering, Biomaterials 2004; 25(19):4749-4757. 
[5] Kaili Lin, Na Zhang, Zhilan Yin, Weibin Zhang, Synthesis of water dispersible silicon containing hydroxyapatite nanoparticles with adjustable degradation rate and their application as $\mathrm{pH}$ responsive drug carriers, China Chemical Engineering Journal, Volume 222, 2013, 49-59.

[6] Omer Kaygili, Sergey V. Dorozhkin, Tankut Ates, N. Canan Gursoy, Serhat Keseer, Fahrettin Yakuphanolgu, A. Birkan Selcuk, Structural and dielectric properties of yttrium- substituted hydroxyapatites, Material Science and Engineering C, 47(2015):333-338.

[7] Masseoud Othmania, Hassen Bachouaa, Yassin Ghandoura, Abdullah Aissaa, Mongi Debbabia, Characterization and catalytic properties of copper-substituted hydroxyapatite nanocrystals, Materials Research Bulletin, 97(2018):560-566.

[8] A.Mariappan, Pandi, N.Balasubramanian, Rajeshwara Palanichamy, K.Neyvasagam, Structural, Optical and Antimicrobial activity of copper and zinc doped Hydroxyapatite nano powders using SolGel method, Material Science \& Engineering, April 2017, ISSN:2412-5954.

[9] Ming li, Pan Xiong, Feng Yan, Sijie Li, Changhong Ren, et al, An overview of graphene-based hydroxyapatite composites fororthopedic applications, Biomaterials, 3(2018):1-18.

[10] Burcin Bazar, Aysen Tezcaner, Dilek Keskin, Zafer Evis, Improvements in microstructural, mechanical and biocompatibility properties of nanosized hydroxyapatites doped with yttrium and fluroide, ceramics international , 36(5), 2010, 1633-1643.

[11] Xue Wei and Matthew Z.Yates, Yttrium doped Hydroxyapatite membranes with high proton conductivity, chemistry of materials, Volume 24, Issue 10, 1738-1743. Publication date (Web): April 27, 2012.

[12] Michiko Sato, Marisa A. Sambito, Arash Aslani, Nader M. Kalkhoran, Elliot B. Slamovich, Thomas Jay Webster, Increased osteoblast functions on undoped and yttrium doped nanocrystalline hydroxyapatite coatings on titanium, 27(11), 2006, 2358-2369. 